\title{
Bioinformatics analysis of hepatic gene expression profiles in type 2 diabetes mellitus
}

\author{
ZHE CHEN $^{1 *}$, WEIQU YUAN ${ }^{2 *}$, TAO LIU ${ }^{3}$, DANPING HUANG $^{2}$ and LEI XIANG ${ }^{4}$ \\ ${ }^{1}$ Institute of Clinical Pharmacology, Guangzhou University of Chinese Medicine, Guangzhou, Guangdong 510405; \\ ${ }^{2}$ The Fourth Clinical Medical School, Guangzhou University of Chinese Medicine, Shenzhen, Guangdong 518033; \\ ${ }^{3}$ School of Traditional Chinese Medicine, Guangdong Pharmaceutical University, Guangzhou, Guangdong 510006; \\ ${ }^{4}$ Department of Integrative Chinese and Western Medicine, The First Affiliated Hospital of Guangdong \\ Pharmaceutical University, Guangzhou, Guangdong 510080, P.R. China
}

Received March 25, 2019; Accepted September 19, 2019

DOI: $10.3892 /$ etm.2019.8092

\begin{abstract}
Type 2 diabetes mellitus (T2DM) is characterized by hyperglycemia. The liver has a critical role in regulating glucose homeostasis. The present study aimed to analyze hepatic gene expression profiles and to identify the key genes and pathways involved in T2DM. Gene expression profiles of 10 patients with T2DM and 7 subjects with normal glucose tolerance were downloaded from the Gene Expression Omnibus database. Subsequently, differentially expressed genes (DEGs) were identified and functional enrichment analysis was performed. In addition, a protein-protein interaction network was built and hub genes were identified. In total, 1,320 DEGs were identified, including 698 up- and 622 downregulated genes, and these were mainly enriched in positive regulation of transcription from RNA polymerase II promoter, cell adhesion, inflammatory response, positive regulation of apoptotic process, signal transduction and the Tolllike receptor signaling pathway. A total of 8 hub genes (G-protein subunit gamma transducin 2, ubiquitinconjugating enzyme E2 D1, glutamate metabotropic receptor 1, G-protein signaling modulator 1, C-X-C motif chemokine ligand 9, neurotensin, purinergic receptor $\mathrm{P} 2 \mathrm{Y} 1$ and ring finger protein 41) were screened from the network. The present study may contribute to the elucidation of the hepatic pathology of T2DM.
\end{abstract}

Correspondence to: Dr Lei Xiang, Department of Integrative Chinese and Western Medicine, The First Affiliated Hospital of Guangdong Pharmaceutical University, 19 Nonglinxia Road, Guangzhou, Guangdong 510080, P.R. China

E-mail: xianglei9527@163.com

${ }^{*}$ Contributed equally

Key words: bioinformatics analysis, hepatic gene expression profiles, type 2 diabetes mellitus, differentially expressed genes, protein-protein interaction network

\section{Introduction}

Type 2 diabetes mellitus (T2DM), resulting from insulin resistance and impaired $\beta$-cell function, constitutes a major health problem throughout the world (1). Exploration of the underlying pathological mechanisms and potential therapeutic targets for T2DM is becoming increasingly important (2).

The liver is involved in glucose metabolism, including gluconeogenesis, glycogenolysis, glycogenesis and insulin extraction (3). Dysregulation of glucose metabolism in the liver contributes to the development of T2DM (4). Disruption in the process of hepatic glucose release gives rise to insulin resistance or diabetes and liver diseases may exacerbate insulin resistance by disturbing the physiological effects of insulin on liver cells (5). A previous study reported that targeted inactivation of the hepatic insulin receptor gene resulted in diabetes-like symptoms, demonstrating a direct involvement of insulin regulation in liver metabolism (6). A further study also revealed that selective inactivation of insulin to disrupt hepatic glucose release and fatty acid synthesis led to insulin resistance in the liver, further corroborating that the liver is a significant target for the effect of insulin (7). Impaired fatty acid metabolism in the liver also causes the development of T2DM (8-10). In addition, a clinical study revealed an elevated incidence of newonset diabetes when patients received liver grafts with steatosis, which is strongly linked to hepatic insulin resistance (11).

Genomic data relevant to various diseases are archived in public repositories that are easily accessed to obtain meaningful information and to make novel discoveries (12). Searching in public repositories has been widely applied to investigate the pathology of T2DM, including the identification of underlying pathways and coexpression networks in islets of patients with T2DM (13-15). The gene expression in the liver of a T2DM mouse model has also been analyzed (16). However, to the best of our knowledge, differentially expressed genes (DEGs) in the liver of T2DM patients vs. subjects with normal glucose tolerance (NGT) have remained to be identified. Therefore, the mechanisms underlying the putative hepatic pathology of T2DM remain to be explored.

In the present study, hepatic DEGs in subjects with T2DM vs. NGT were identified, and subsequently, functional 
enrichment analysis was performed. A protein-protein interaction (PPI) network was also built to identify hub genes. The results of the present study may contribute towards the elucidation of the hepatic pathology of T2DM.

\section{Materials and methods}

Microarray data. A gene expression profile (accession no. GSE23343) was obtained from the Gene Expression Omnibus (GEO; http://www.ncbi.nlm.nih.gov/geo/). The GEO database stores abundant highthroughput data, particularly those generated by DNA microarray technology (17). A total of 10 patients ( 6 males and 4 females) with T2DM and 7 subjects (4 males and 3 females) with NGT were included in this GEO dataset, and their clinical characteristics are available from the supplementary information online (18). The array data were acquired from the Affymetrix Human Genome U133 Plus 2.0 array [GPL570; transcript (gene) version].

DEG analysis. The gene expression profiles of liver samples from subjects with T2DM and NGT in the dataset GSE23343 were compared to identify DEGs. This analysis was performed using GEO2R (http://www.ncbi.nlm.nih.gov/geo/geo2r/) through Rbased analysis of the microarray data (12). Ilog (fold change) $\mid \geq 1$ and $\mathrm{P}<0.05$ were the cut-off criteria. A heatmap of these DEGs was drawn using MeV 4.9.0 (https://sourceforge. net/projects/mevtm4/).

Enrichment analysis. Gene Ontology (GO) and Kyoto Encyclopedia of Genes and Genomes (KEGG) pathway enrichment analysis of the DEGs were performed using the Database of Annotation Visualization and Integrated Discovery (DAVID 6.8; https://david.ncifcrf.gov/) (19). The GO categories were biological process (BP), molecular function $(\mathrm{MF})$ and cellular component $(\mathrm{CC}) . \mathrm{P}<0.05$ was considered to indicate a statistically significant difference. The results of the enrichment analysis were visualized in a bubble chart using the OmicShare tools 3.0, a free online platform for data analysis and visualization (http://www.omicshare.com/tools).

PPI network analysis. The Search Tool for the Retrieval of Interacting Genes (STRING 10.5; https://stringdb.org/) was used to construct a PPI network. This website offers predicted and verified interactions among numerous proteins (20). A combined score $>0.7$ was selected as the cutoff criterion. Subsequently, the screened PPI network was imported into Cytoscape3.2.1 (http://www.cytoscape.org/) to identify critical gene modules and hub genes. Nodes with a high degree ( $\geq 2$ fold the median number of connections with other nodes) were considered as significant nodes and nodes with a higher degree ( $\geq 5$ fold the median number of connections with other nodes) were considered as hub nodes. Submodules of the network were screened using Molecular Complex Detection (MCODE 1.4.2) (21), with the criteria of node number $>10$ and MCODE score $>10$. Finally, enrichment analysis of the submodules was performed using DAVID.

\section{Results}

DEG analysis. A total of 1,320 DEGs in liver samples of patients with T2DM vs. NGT samples were identified, including
698 up- and 622 downregulated genes. The heat-map of the top 50 up- and top 50 downregulated genes is presented in Fig. 1.

GO analysis. In the $\mathrm{GO}$ category $\mathrm{BP}$, upregulated genes were mainly enriched in positive regulation of transcription from RNA polymerase II (RNAP II) promoter, cell adhesion, inflammatory response, positive regulation of apoptotic process and extracellular matrix organization (Table I), whereas downregulated genes were mainly associated with signal transduction, multicellular organism development, positive regulation of GTPase activity, visual perception and axon guidance (Table II). In the GO category MF, upregulated genes were mainly involved in calcium ion binding, extracellular matrix structural constituent, SMAD binding, Rho guanylnucleotide exchange factor activity and 3',5'-cyclic AMP phosphodiesterase activity (Table I), whereas downregulated genes were mainly involved in actin binding, receptor activity, RNAP II transcription factor activity, sequence-specific DNA binding, calmodulin binding and protein tyrosine kinase activity (Table II). Finally, concerning the GO category $\mathrm{CC}$, upregulated genes were mainly involved in the plasma membrane, integral component of plasma membrane, extracellular region, cell junction and cytoskeleton (Table I), whereas downregulated genes were mainly involved in nuclear envelope, myosin complex, microvillus growth cone membrane and actomyosin (Table II).

KEGG pathway analysis. Upregulated genes were mainly enriched in transcriptional misregulation in cancer, Toll-like receptor (TLR) signaling pathway, inflammatory mediator regulation of transient receptor potential (TRP) channels, glutamatergic synapse, and protein digestion and absorption, whereas downregulated genes were mainly associated with endocytosis, tight junction and melanoma (Table II). The results of the enrichment analysis were visualized in Figs. 2 and 3, respectively.

PPI network analysis. As presented in Fig. 4, the PPI network of DEGs consisted of 443 nodes and 996 edges. A total of 11 genes were selected as candidates for hub genes. In addition, two submodules were selected, one of which had 28 nodes and 197 edges, while the other module had 14 nodes and 91 edges (Fig. 5). Finally, as presented in Table III, eight hub genes involved in these two submodules were identified, including Gprotein subunit gamma transducin 2 (GNGT2), ubiquitin-conjugating enzyme E2 D1 (UBE2D1), glutamate metabotropic receptor 1 (GRM1), G-protein signaling modulator 1 (GPSM1), C-X-C motif chemokine ligand 9 (CXCL9), neurotensin (NTS), purinergic receptor P2Y1 (P2RY1) and ring finger protein 41 (RNF41). However, no enrichment was observed in these two submodules.

\section{Discussion}

In the present study, 698 up- and 622 downregulated DEGs were screened from the hepatic genes of patients with T2DM and normal subjects. GO term analysis revealed that the upregulated DEGs were mainly associated with positive regulation of transcription from RNAP II promoter, cell adhesion, inflammatory response, positive regulation of apoptotic 
$-1.0$

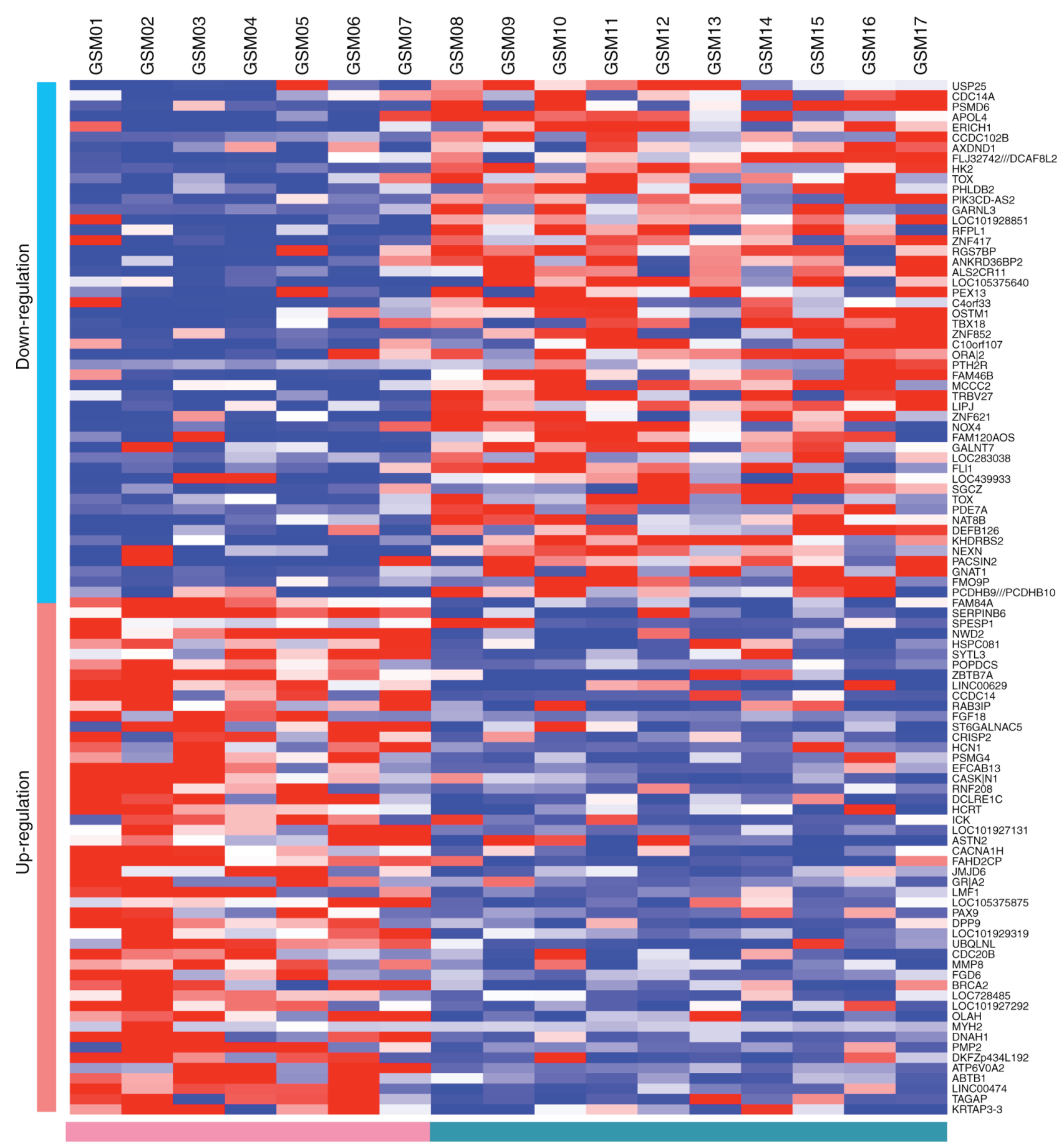

Normal glucose tolerance

Type 2 diabetes mellitus

Figure 1. Heat-map of the top 50 up- and top 50 downregulated DEGs in T2DM $(\mathrm{P}<0.05)$. The red color represents a higher expression value, whereas the blue color represents a lower expression value. DEG, differentially expressed gene; T2DM, type 2 diabetes mellitus.

process and extracellular matrix organization. Hepatocyte nuclear factor 4 (HNF4) regulates numerous pivotal metabolic pathways and exert significant effects on recruiting RNAP II to synthesize gene promoters. Abnormalities in the hepatic HNF4 transcription network are accountable for diabetes and fatty liver (22). Alterations in cell adhesion may disturb significant cellular processes, leading to the causation of various diseases. Targeted inactivation of carcinoembryonic antigen-related cell adhesion molecule 1 (CEACAM1) in the liver was reported to cause insulin resistance and promote hepatic adipogenesis, suggesting a critical role of CEACAM1 in regulating insulin clearance in the liver (23). Hyperglycemiainduced oxidative stress induces liver tissue injury and the ensuing derangement of protein, carbohydrate and lipid metabolism leads to increased oxidative stress, further triggering the inflammatory cascade (24). Hepatocyte inflammation significantly downregulates insulin signaling components, including insulin receptor substrate (IRS)-1, IRS-2, PI3K, Akt and 
Table I. GO analysis of up- and downregulated genes in type 2 diabetes mellitus $(\mathrm{P}<0.05)$.

A, Upregulation

Category/term

N $(\%) \quad$ P-value

$\mathrm{BP}$

GO:0045944-Positive regulation of transcription from RNA polymerase II promoter

$\begin{array}{ll}39(5.972) & 0.049 \\ 23(3.522) & 0.017 \\ 20(3.063) & 0.017 \\ 17(2.603) & 0.016 \\ 16(2.450) & 0.001\end{array}$

GO:0007155-Cell adhesion

GO:0006954-Inflammatory response

GO:0043065-Positive regulation of apoptotic process

GO:0030198-Extracellular matrix organization

$16(2.450) \quad 0.001$

$\mathrm{CC}$

GO:0005886-Plasma membrane

$151(23.124) \quad 0.001$

GO:0005887-Integral component of plasma membrane

$64(9.801) \quad 0.000$

GO:0005576-Extracellular region

$59(9.035) \quad 0.049$

GO:0030054-Cell junction

$24(3.675) \quad 0.008$

GO:0005856-Cytoskeleton

$19(2.910) \quad 0.024$

$\mathrm{MF}$

GO:0005509-Calcium ion binding

$31(4.747) \quad 0.033$

GO:0005201-Extracellular matrix structural constituent

$8(1.225) \quad 0.003$

GO:0046332-SMAD binding

$7(1.072) \quad 0.001$

GO:0005089-Rho guanyl-nucleotide exchange factor activity

$7(1.072) \quad 0.025$

GO:0004115-3',5'-cyclic-AMP phosphodiesterase activity

$4(0.613)$

0.009

B, Downregulation

Category/term

$\mathrm{N}(\%)$

P-value

BP

GO:0007165-Signal transduction

GO:0007275-Multicellular organism development

$46(7.931) \quad 0.002$

GO:0043547-Positive regulation of GTPase activity

$25(4.310) \quad 0.003$

GO:0007601-Visual perception

$25(4.310) \quad 0.007$

GO:0007411-Axon guidance

$12(2.069) \quad 0.011$

$\mathrm{CC}$

GO:0005635-Nuclear envelope

$9(1.552) \quad 0.043$

GO:0016459-Myosin complex

$9(1.552) \quad 0.039$

GO:0005902-Microvillus

$5(0.862) \quad 0.032$

GO:0032584-Growth cone membrane

$5(0.862)$

0.049

GO:0042641-Actomyosin

$3(0.517)$

0.011

$3(0.517)$

0.033

$\mathrm{MF}$

GO:0003779-Actin binding

$14(2.414) \quad 0.022$

GO:0004872-Receptor activity

$12(2.069)$

0.020

GO:0000981-RNA polymerase II transcription factor activity, sequence-specific DNA binding

$11(1.897)$

0.010

GO:0005516-Calmodulin binding

$11(1.897) \quad 0.020$

GO:0004713-Protein tyrosine kinase activity

$10(1.724)$

0.006

BP, biological process; CC, cellular component; MF, molecular function; GO, gene ontology.

mTOR (25). Inflammatory regulators induced by hepatocyte apoptosis-associated damage are able to regulate the insulin signaling pathway, and these insulin resistanceassociated regulators may, in turn, affect hepatocyte apoptosis (5).
Endoplasmic reticulum stressinduced apoptosis of hepatocytes and adipocytes is also important in the development of diabetes, characterized by increased insulin resistance (26). The downregulated DEGs were mainly involved in signal transduction, 
Table II. Kyoto Encyclopedia of Genes and Genomes pathway analysis of up- and downregulated genes in type 2 diabetes mellitus $(\mathrm{P}<0.05)$.

A, Upregulated genes

\begin{tabular}{|c|c|c|c|}
\hline Term & $\mathrm{N}(\%)$ & P-value & Genes \\
\hline hsa05202: Transcriptional misregulation in cancer & $11(1.685)$ & 0.039 & $\begin{array}{l}\text { MAX, CD86, FLI1, SP1, CCND2, PML, ETV1, } \\
\text { MDM2, JMJD1C, ETV5, MYCN }\end{array}$ \\
\hline hsa04620: Toll-like receptor signaling pathway & $10(1.531)$ & 0.006 & $\begin{array}{l}\text { IFNA2, CD86, IFNA7, MAPK14, CXCL9, } \\
\text { MAPK10, CXCL11, TLR6, TLR8, SPP1 }\end{array}$ \\
\hline $\begin{array}{l}\text { hsa04750: Inflammatory mediator regulation of } \\
\text { TRP channels }\end{array}$ & $9(1.378)$ & 0.012 & $\begin{array}{l}\text { PRKCQ, PLA2G4A, IL1R1, PTGER4, MAPK14, } \\
\text { F2RL1, MAPK10, HTR2B, PRKCB }\end{array}$ \\
\hline hsa04724: Glutamatergic synapse & $9(1.378)$ & 0.027 & $\begin{array}{l}\text { SLC17A8, PLA2G4A, GNGT2, GRIK1, GRIN1, } \\
\text { SLC38A1, GRM1, SHANK2, PRKCB }\end{array}$ \\
\hline hsa04974: Protein digestion and absorption & $8(1.225)$ & 0.021 & $\begin{array}{l}\text { SLC8A1, COL14A1, COL13A1, PRCP, COL1A2, } \\
\text { COL12A1, ATP1A1, COL5A2 }\end{array}$ \\
\hline
\end{tabular}

B, Downregulated genes

\begin{tabular}{lcrl}
\hline Term & Count & P-value & \\
\hline hsa04144: Endocytosis & $12(2.069)$ & 0.031 & $\begin{array}{l}\text { ARFGAP1, IGF1R, CBLC, RET, PIP5KL1, } \\
\text { FOLR1, SNX5, RAB35, KIF5C, CYTH4, GRK4, } \\
\text { DNM1 }\end{array}$ \\
hsa04530: Tight junction & $8(1.379)$ & 0.035 & $\begin{array}{l}\text { SHROOM4, MYH2, EXOC4, MYH14, MYH8, } \\
\text { CLDN23, MYH7B, AKT2 }\end{array}$ \\
hsa05218: Melanoma & $6(1.034)$ & 0.022 & FGF6, IGF1R, FGF18, CDKN2A, AKT2, FGF4 \\
\hline
\end{tabular}

Hsa, Homo sapiens; TRP, transient receptor potential; MAX, myc-associated factor X; CD86, T-lymphocyte activation antigen CD86; FLI1, friend leukemia integration 1 transcription factor; SP1, transcription factor sp1; CCND2, cyclin-D2; PML, promyelocytic leukemia; ETV1, ETS translocation variant 1; MDM2, E3 ubiquitin-protein ligase mdm2; JMJD1C, jumonji domain-containing protein 1C; ETV5, ETS variant transcription factor 5; MYCN, N-myc proto-oncogene; IFNA2, interferon $\alpha$-2; IFNA7, interferon $\alpha-7$; MAPK14, mitogen-activated protein kinase 14; CXCL9, C-X-C motif chemokine 9; MAPK10, mitogen-activated protein kinase 10; CXCL11, C-X-C motif chemokine 11; TLR6, Toll-like receptor 6; TLR8, Toll-like receptor 8; SPP1, secreted phosphoprotein 1; PRKCQ, protein kinase C $\theta$; PLA2G4A, phospholipase A2 group IVA; IL1R1, interleukin-1 receptor type 1; PTGER4, prostaglandin E receptor 4; F2RL1, coagulation factor II receptor-like 1; HTR2B, 5-hydroxytryptamine receptor 2B; PRKCB, protein kinase C $\beta$; SLC17A8, solute carrier family 17 member 8; GNGT2, G protein subunit $\gamma$ transducin 2; GRIK1, glutamate receptor ionotropic kainate 1; GRIN1, glutamate receptor ionotropic NMDA 1; SLC38A1, solute carrier family 38 member 1; GRM1, glutamate metabotropic receptor 1; SHANK2, SH3 and multiple ankyrin repeat domains 2; SLC8A1, solute carrier family 8 member A1; COL14A1, collagen type XIV $\alpha 1$ chain; COL13A1, collagen type XIII $\alpha 1$ chain; PRCP, prolylcarboxypeptidase; COL1A2, collagen type I $\alpha 2$ chain; COL12A1, collagen type XII $\alpha 1$ chain; ATP1A1, ATPase Na+/K+ transporting subunit $\alpha$ 1; COL5A2, collagen type V $\alpha 2$ chain; ARFGAP1, ADP-ribosylation factor GTPase-activating protein 1; IGF1R, insulin-like growth factor 1 receptor; CBLC, E3 ubiquitin-protein ligase CBL-C; RET, ret proto-oncogene; PIP5KL1, phosphatidylinositol-4-phosphate 5-kinase like 1; FOLR1, folate receptor $\alpha$; SNX5, Sorting nexin-5; RAB35, Ras-related protein Rab-35; KIF5C, kinesin family member 5C; CYTH4, Cytohesin-4; GRK4, G protein-coupled receptor kinase 4; DNM1, dynamin 1; SHROOM4, shroom family member 4; MYH2, myosin heavy chain 2; EXOC4, exocyst complex component 4; MYH14, myosin heavy chain 14; MYH8, myosin heavy chain 8; CLDN23, Claudin-23; MYH7B, myosin-7B; AKT2, RAC- $\beta$ serine/threonine-protein kinase; FGF6, fibroblast growth factor 6; FGF18, fibroblast growth factor 18; CDKN2A, cyclin dependent kinase inhibitor 2A; FGF4 fibroblast growth factor 4.

multicellular organism development and positive regulation of GTPase activity. In the process of metabolic alterations, cellular responses to extracellular stimulation require signal transduction, contributing to physiological events including increased uptake of blood glucose (27). GTPases are also important in signal transduction at the intracellular domain of transmembrane receptors (28).

The KEGG pathway enrichment analysis indicated that the upregulated DEGs were accumulated in the TLR signaling pathway, inflammatory mediator regulation of TRP channels and protein digestion and absorption, and that the downregulated DEGs were enriched in endocytosis and tight junction. Diabetes frequently occurs in combination with other metabolic diseases, including hyperlipidemia, hypertension and non-alcoholic fatty liver disease (29). Deposition of fatty acids in the liver, particularly saturated fatty acids, activates the TLR pathway, which is associated with the inflammatory response (30). Hepatic inflammation is closely correlated with insulin resistance (25). A previous study reported that TRP cation channel subfamily $\mathrm{V}$ member 4 effectively 


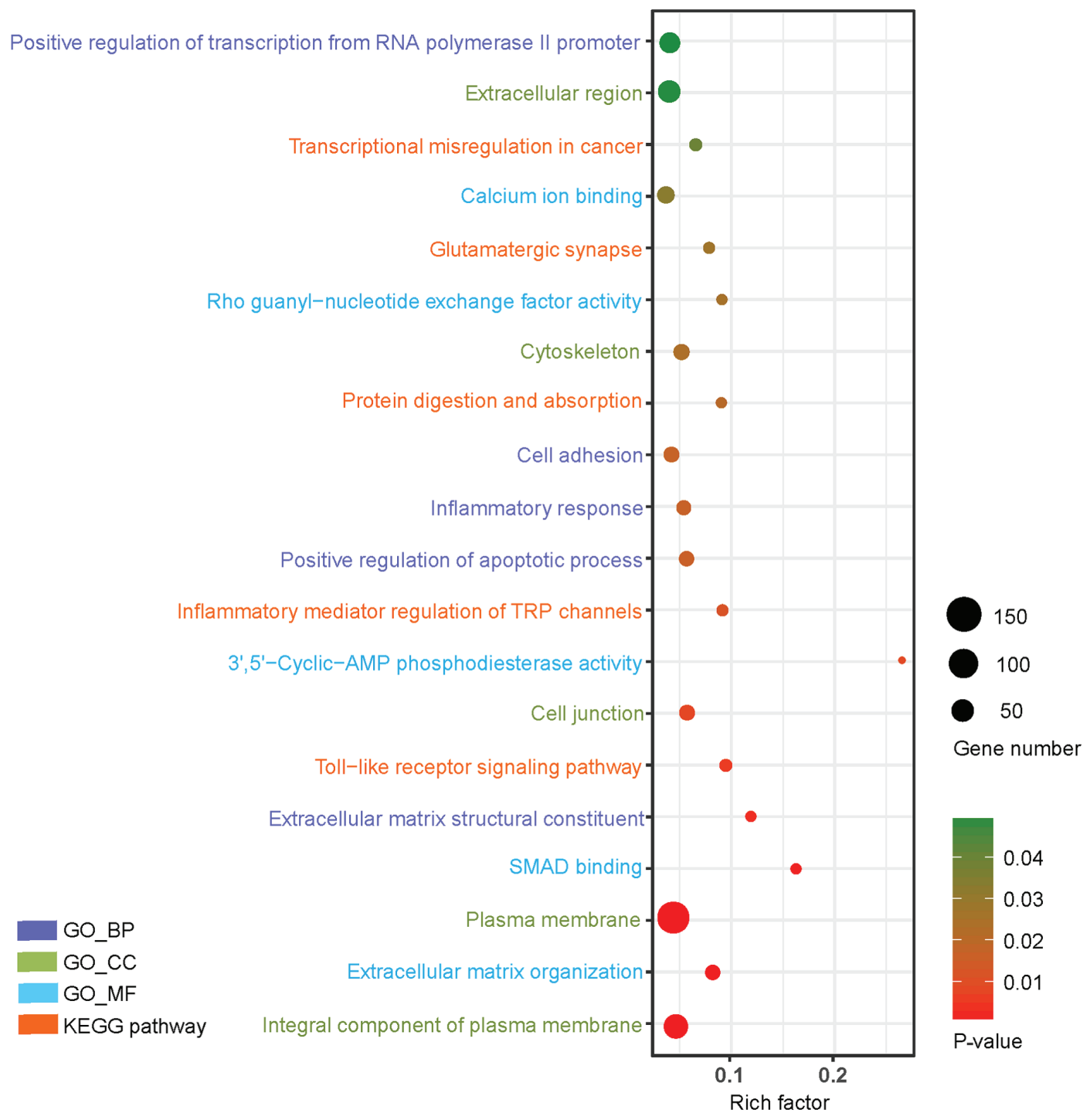

Figure 2. GO and KEGG pathway analysis of the top 20 upregulated genes in type 2 diabetes mellitus $(\mathrm{P}<0.05)$. The $\mathrm{Y}$-axis represents GO categories, including BP, MF and CC, whereas the Xaxis represents the enrichment factor. The enrichment factor is the ratio of the number of DEGs annotated to the term to the number of all genes annotated to it. In addition, the dot size represents the number of DEGs annotated to the term, whereas the dot color indicates the significance of gene enrichment. GO, gene ontology; KEGG, Kyoto Encyclopedia of Genes and Genomes; DEG, differentially expressed gene; TRP, transient receptor potential; BP, biological process; CC, cellular component; MF, molecular function.

regulates the expression of various pro-inflammatory genes in adipose tissue, and that these pro-inflammatory genes are closely associated with insulin resistance (31). Tight-junction proteins, besides their function as integral proteins of tight junctions that form barriers in the gut and the liver, may also be expressed outside the tight junction to regulate signaling, trafficking and gene expression. A hallmark is their regulation of epithelial-to-mesenchymal transition (32). A previous study demonstrated that the endocytosis impairment of specific ligands or other macromolecules may represent an important pathology mechanism in diabetes (33). The biological processes and pathways identified and discussed above may indicate an important role of the liver in the pathology of T2DM.

In the present study, the following eight hub genes were also selected: GNGT2, UBE2D1, GRM1, GPSM1, CXCL9, NTS, P2RY1 and RNF41. GNGT2 was reported to be involved in $\beta$-arrestin-1induced Akt phosphorylation and NF- $\mathrm{KB}$ activation (34). Activation of $\mathrm{NF \kappa B}$ in the liver may result in hepatic insulin resistance (5). The low-density lipoprotein (LDL) receptor is indispensable for the uptake of LDL cholesterol and for regulating the levels of plasma lipoprotein (35). The E3 ubiquitin ligase inducible degrader of LDL receptor/ubiquitin-conjugating enzyme E2D complex is effectively responsible for determining LDL receptor activity (36). Sirtuin 1, a type of nicotinamide adenine dinucleotide-dependent deacetylase, also regulates the pathogenesis of metabolic disease, aging and tumorigenesis (37). Sirtuin 1-mediated epigenetic regulation of the expression of the metabotropic glutamate receptor $1 / 5$ (encoded by the GRM1/5 gene) was reported to be involved in the development of neuropathic pain in a rat model of T2DM $(38,39)$. The GPSM1 locus has been demonstrated to be associated with the insulinogenic index and with the fasting glucose level $(40,41)$, and GPSM1 has been identified as one of the three novel T2DM loci in East Asian populations (42). Previous studies have also suggested an important role of CXCL9 in diabetic neuropathy, diabetic 
Table III. Hub nodes in the network of differentially expressed genes in type 2 diabetes mellitus.

\begin{tabular}{|c|c|c|c|c|c|}
\hline Hub node & Description & Degree & MCODE score & Count & Up/downregulation \\
\hline GNGT2 & G protein subunit gamma transducin 2 & 39 & 14 & 41 & Up \\
\hline UBE2D1 & Ubiquitin-conjugating enzyme E2 D1 & 19 & 13 & 29 & Up \\
\hline GRM1 & Glutamate metabotropic receptor 1 & 17 & 13 & 20 & $\mathrm{Up}$ \\
\hline GPSM1 & G-protein signaling modulator 1 & 16 & 14 & 18 & Down \\
\hline CXCL9 & $\mathrm{C}-\mathrm{X}-\mathrm{C}$ motif chemokine ligand 9 & 16 & 14 & 24 & Up \\
\hline NTS & Neurotensin & 16 & 13 & 38 & Up \\
\hline P2RY1 & Purinergic receptor P2Y1 & 16 & 13 & 17 & Up \\
\hline RNF41 & Ring finger protein 41 & 16 & 13 & 17 & Up \\
\hline
\end{tabular}

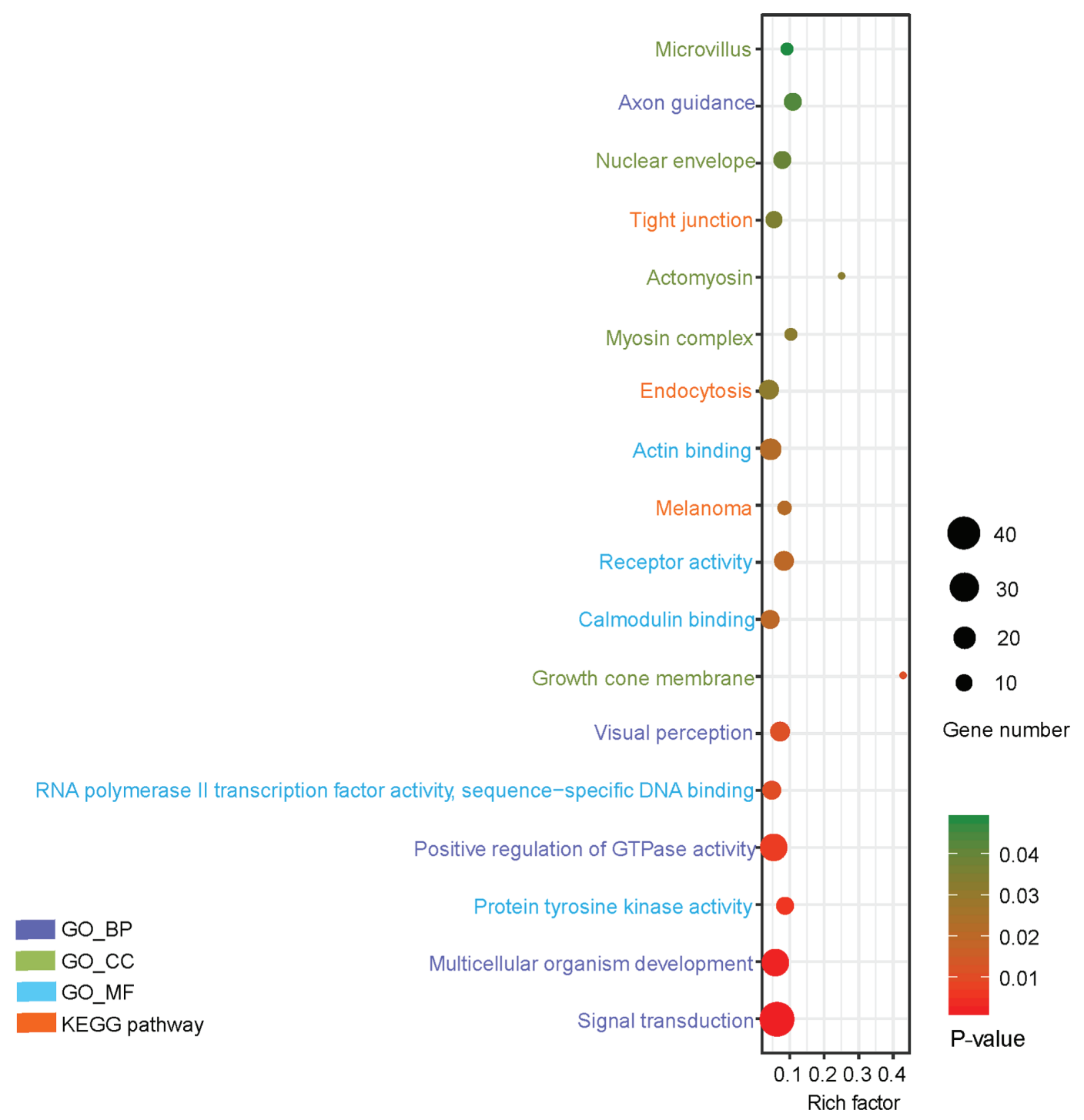

Figure 3. GO and KEGG pathway analysis of the top 18 downregulated genes in type 2 diabetes mellitus (P<0.05). The Y-axis represents GO categories, including BP, MF and CC, whereas the X-axis represents the enrichment factor. The enrichment factor is the ratio of the number of DEGs annotated to the term to all the genes annotated to it. In addition, the dot size represents the number of DEGs annotated to the term, whereas the dot color indicates the significance of gene enrichment. GO, gene ontology; KEGG, Kyoto Encyclopedia of Genes and Genomes; DEG, differentially expressed gene; BP, biological process; $\mathrm{CC}$, cellular component; MF, molecular function.

retinopathy and diabetic nephropathy (43-45). Advanced glycation end products were reported to promote apoptosis and inflammation in mouse podocytes via CXCL9-regulated activation of the JAK2/STAT3 pathway (46). The fasting plasma levels of pro-NTS produced in equimolar amounts with NTS were indicated to be positively associated with the risk of 


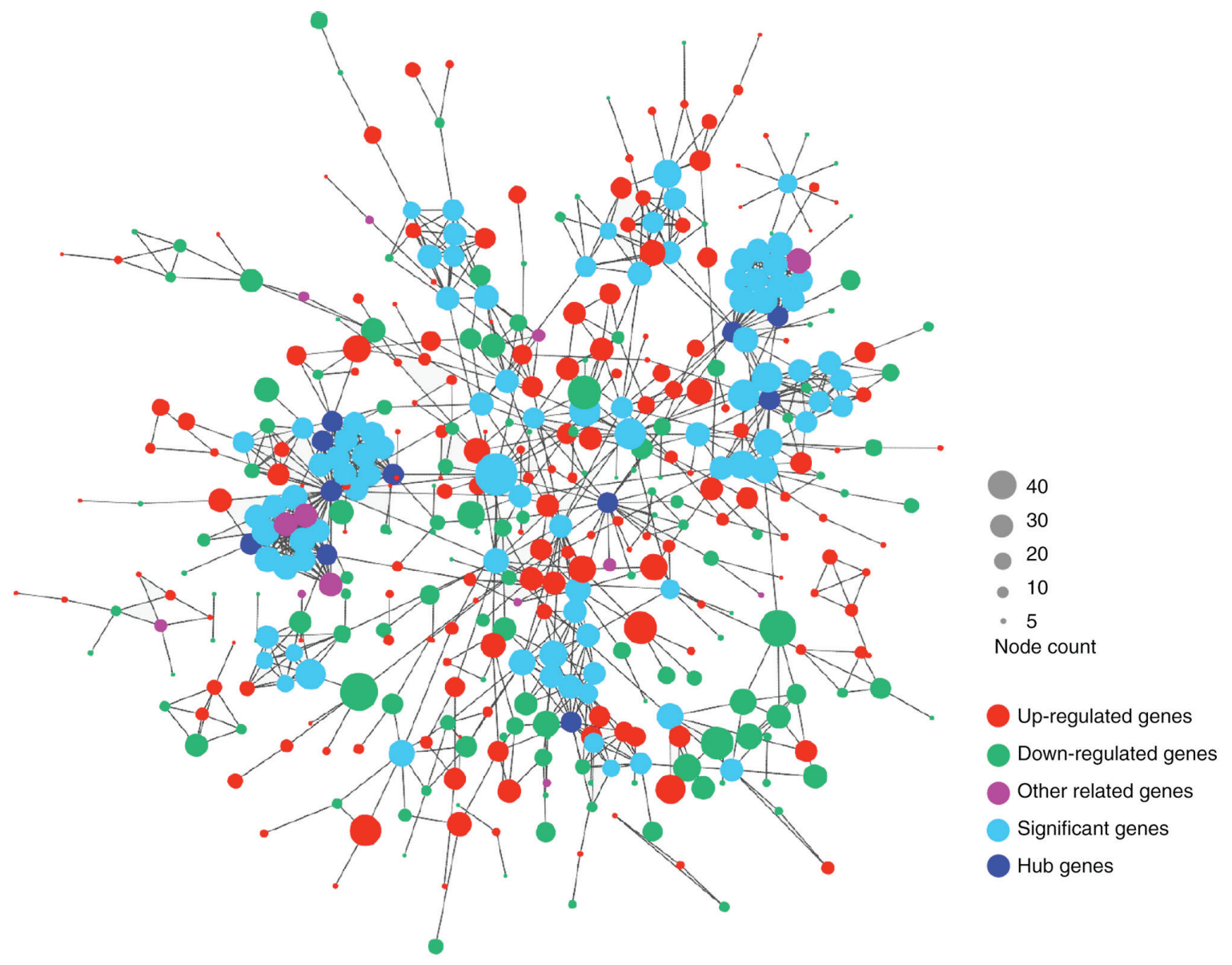

Figure 4. Protein-protein interaction network of the DEGs in type 2 diabetes mellitus. The red nodes represent the upregulated genes, whereas green nodes represent the downregulated genes. Specifically, light blue nodes indicate the significant genes with a high degree $(\geq 2$-fold the median number of connections with other nodes), whereas dark blue nodes indicate the hub genes with a higher degree ( $\geq 5$-fold the median number of connections with other nodes). In addition, purple nodes indicate other genes associated with the DEGs that were identified. The node size indicates the node counts. DEG, differentially expressed gene.
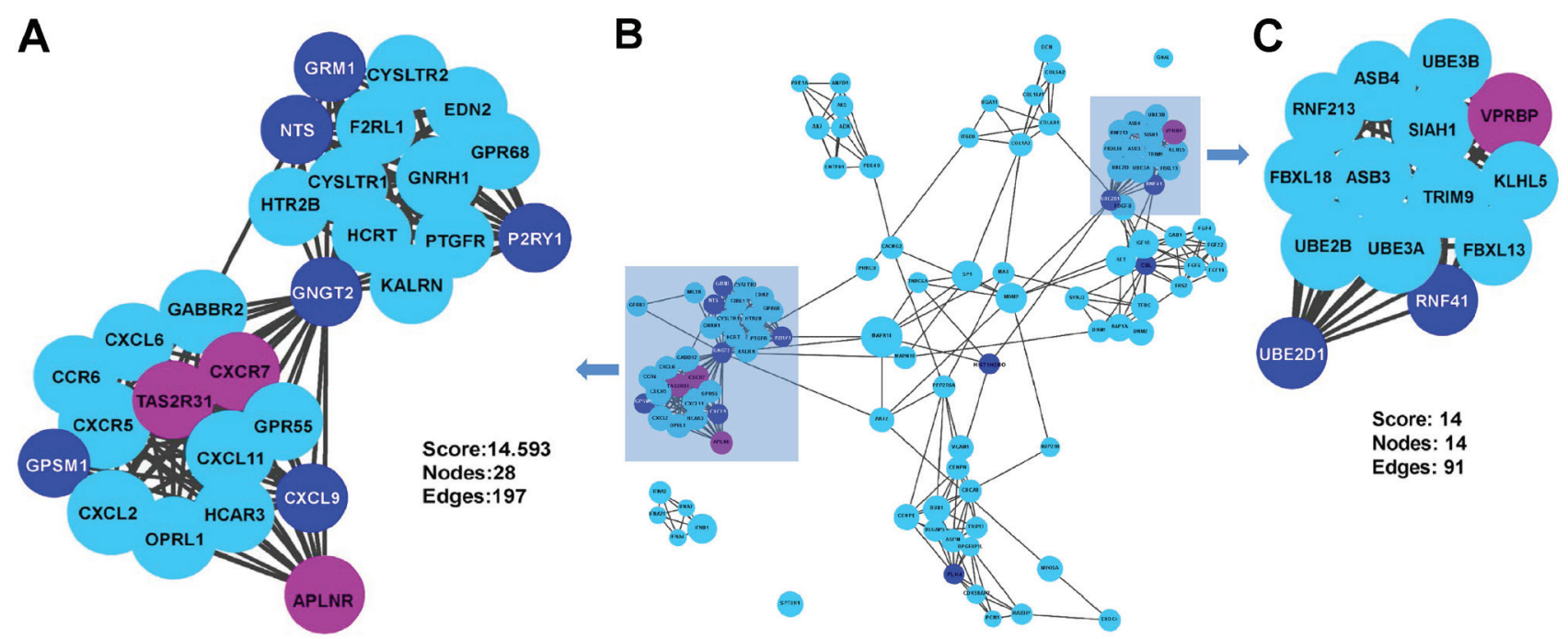

Figure 5. (A) One of the two significant submodules. (B) Core protein-protein interaction network of differentially expressed genes in type 2 diabetes mellitus. (C) The other significant submodule.

diabetes, cardiovascular disease and mortality (47). Obese and insulin-resistant patients had higher plasma concentrations of proNTS, and NTS-deficient mice on a highfat diet exhibited lower levels of fasting plasma glucose and insulin compared with wildtype mice (48). Furthermore, P2Y1 receptorknockout mice exhibited high levels of plasma insulin, plasma glucose 
and increased body weight, indicating an important regulatory role of the P2Y1 receptors in glucose homeostasis (49). Finally, RNF41, an E3 ubiquitin ligase, was identified to be essential for activation of the type 1 interferon pathway to sustain insulin sensitivity in the muscle tissue of obese patients (50). Pancreatic islet $\beta$-cells require normal mitochondrial function in terms of their high metabolic activity. Stabilization of the C-type lectin domain-containing 16A/RFP41/ubiquitin-specific peptidase 8 mitochondrial autophagy complex is essential for cellular respiration and insulin secretion. However, a study reported that elevated levels of glucose and fatty acids destabilized the complex, causing $\beta$-cell apoptosis (51).

In conclusion, a comprehensive analysis of hepatic DEGs in T2DM was performed in the present study, revealing an important role of the liver in the pathological mechanisms of T2DM. However, considering the absence of clinical validation in the present study, further investigation of these mechanisms underlying the hepatic pathology of T2DM is required to confirm these results.

\section{Acknowledgements}

Not applicable.

\section{Funding}

The present study was supported by the National Natural Science Foundation of China (grant no. 81804030).

\section{Availability of data and materials}

All data can be accessed in the GEO database (https://www. ncbi.nlm.nih.gov/geo/query/acc.cgi?acc=GSE23343).

\section{Authors' contributions}

ZC and LX designed the study. ZC, WY, TL and DH performed the data analysis. ZC, TL and DH drafted the manuscript. ZC, WY and LX revised the manuscript. All authors agree to be accountable for all aspects of the work and gave approval for the study to be published.

\section{Ethics approval and consent to participate}

Not applicable.

\section{Patient consent for publication}

Not applicable.

\section{Competing interests}

The authors declare that they have no competing interests.

\section{References}

1. American Diabetes Association: Diagnosis and Classification of Diabetes Mellitus. Diabetes Care 32 (Suppl 1): S62-S67, 2009.

2. Li L, Pan Z, Yang S, Shan W and Yang Y: Identification of key gene pathways and coexpression networks of islets in human type 2 diabetes. Diabetes Metab Syndr Obes 11: 553-563, 2018.
3. Ling $\mathrm{Q}, \mathrm{Xu} \mathrm{X}$, Wang $\mathrm{B}, \mathrm{Li} \mathrm{L}$ and Zheng S: The origin of new-onset diabetes after liver transplantation: Liver, Islets, or Gut? Transplantation 100: 808-813, 2016

4. DeFronzo RA: Pathogenesis of type 2 diabetes mellitus. Med Clin North Am 88: 787-835, 2004.

5. Schattenberg JM and Schuchmann M: Diabetes and apoptosis: Liver. Apoptosis 14: 1459-1471, 2009.

6. Michael MD, Kulkarni RN, Postic C, Previs SF, Shulman GI, Magnuson MA and Kahn CR: Loss of insulin signaling in hepatocytes leads to severe insulin resistance and progressive hepatic dysfunction. Mol Cell 6: 87-97, 2000.

7. Shimomura I, Matsuda M, Hammer RE, Bashmakov Y, Brown MS and Goldstein JL: Decreased IRS-2 and increased SREBP-1c lead to mixed insulin resistance and sensitivity in livers of lipodystrophic and ob/ob Mice. Mol Cell 6: 77-86, 2000.

8. Lewis GF, Carpentier A, Adeli K and Giacca A: Disordered fat storage and mobilization in the pathogenesis of insulin resistance and type 2 diabetes. Endocr Rev 23: 201-229, 2002.

9. McGarry JD: Banting lecture 2001 dysregulation of fatty acid metabolism in the etiology of type 2 diabetes. Diabetes 51: 7-18, 2002.

10. Postic C and Girard J: Contribution of de novo fatty acid synthesis to hepatic steatosis and insulin resistance: Lessons from genetically engineered mice. J Clin Invest 118: 829-838, 2008.

11. Honda M, Asonuma K, Hayashida S, Suda H, Ohya Y, Lee KJ, Yamamoto $\mathrm{H}$, Takeichi $\mathrm{T}$ and Inomata $\mathrm{Y}$ : Incidence and risk factors for new-onset diabetes in living-donor liver transplant recipients. Clin Transplant 27: 426-435, 2013.

12. Barrett T, Wilhite SE, Ledoux P, Evangelista C, Kim IF, Tomashevsky M, Marshall KA, Phillippy KH, Sherman PM, Holko M, et al: NCBI GEO: Archive for functional genomics data sets-update. Nucleic Acids Res 41 (Databaseissue): D991-D995, 2013.

13. Gaulton KJ,Ferreira T, Lee Y, Raimondo A, Mägi R, Reschen ME, Mahajan A, Locke A, Rayner NW, Robertson N, et al: Genetic fine mapping and genomic annotation defines causal mechanisms at type 2 diabetes susceptibility loci. Nat Genet 47: 1415-1425, 2015.

14. Lawlor N, Khetan S, Ucar D and Stitzel ML: Genomics of Islet (Dys)function and Type 2 Diabetes. Trends Genet 33: 244-255, 2017.

15. Stitzel ML, Sethupathy P, Pearson DS, Chines PS, Song L, Erdos MR, Welch R, Parker SC, Boyle AP, Scott LJ, et al: Global epigenomic analysis of primary human pancreatic islets provides insights into type 2 diabetes susceptibility loci. Cell Metab 12: 443-455, 2010

16. Zhang F, Xu X, Zhang Y, Zhou B, He Z and Zhai Q: Gene expression profile analysis of type 2 diabetic mouse liver. PLoS One 8: e57766, 2013

17. Barrett T and Edgar R: Gene expression omnibus: Microarray data storage, submission, retrieval, and analysis. Methods Enzymo 411: 352-369, 2006.

18. Misu H, Takamura T, Takayama $H$, Hayashi $H$, Matsuzawa-Nagata N, Kurita S, Ishikura K, Ando H, Takeshita Y, Ota T, et al: A liver-derived secretory protein, selenoprotein $\mathrm{P}$, causes insulin resistance. Cell Metab 12: 483-495, 2010.

19. Dennis G Jr, Sherman BT, Hosack DA, Yang J, Gao W, Lane HC and Lempicki RA: DAVID: Database for annotation, visualization, and integrated discovery. Genome Biol 4: P3, 2003.

20. Szklarczyk D, Franceschini A, Kuhn M, Simonovic M, Roth A, Minguez P, Doerks T, Stark M, Muller J, Bork P, et al: The STRING database in 2011: Functional interaction networks of proteins, globally integrated and scored. Nucleic Acids Res 39 (Database): 561-568, 2011

21. Bader GD and Hogue CW: An automated method for finding molecular complexes in large protein interaction networks. BMC Bioinformatics 4: 2, 2003.

22. Rana R, Surapureddi S, Kam W, Ferguson S and Goldstein JA: Med25 is required for RNA polymerase II recruitment to specific promoters, thus regulating xenobiotic and lipid metabolism in human liver. Mol Cell Biol 31: 466-481, 2011.

23. DeAngelis AM, Heinrich G, Dai T, Bowman TA, Patel PR, Lee SJ, Hong EG, Jung DY, Assmann A, Kulkarni RN, et al: Carcinoembryonic antigen-related cell adhesion molecule 1: A link between insulin and lipid metabolism. Diabetes 57: 2296-2303, 2008.

24. Mohamed J, Nazratun Nafizah AH, Zariyantey AH and Budin SB: Mechanisms of diabetes-induced liver damage: The role of oxidative stress and inflammation. Sultan Qaboos Univ Med J 16: e132-e141, 2016. 
25. Sadi G, Pektas MB, Koca HB, Tosun M and Koca T: Resveratrol improves hepatic insulin signaling and reduces the inflammatory response in streptozotocin-induced diabetes. Gene 570: 213-220, 2015.

26. van der Kallen CJ, van Greevenbroek MM, Stehouwer CD and Schalkwijk CG: Endoplasmic reticulum stress-induced apoptosis in the development of diabetes: Is there a role for adipose tissue and liver? Apoptosis 14: 1424-1434, 2009.

27. Rosen OM: After insulin binds. Science 237: 1452-1458, 1987.

28. Scheffzek K and Ahmadian MR: GTPase activating proteins: Structural and functional insights 18 years after discovery. Cell Mol Life Sci 62: 3014-3038, 2005.

29. Wang H, Zhang Q, Chai Y, Liu Y, Li F, Wang B, Zhu C, Cui J, $\mathrm{Qu} \mathrm{H}$ and Zhu M: 1,25(OH)2D3 downregulates the Toll-like receptor 4-mediated inflammatory pathway and ameliorates liver injury in diabetic rats. J Endocrinol Invest 38: 1083-1091, 2015.

30. Huang S, Rutkowsky JM, Snodgrass RG, Ono-Moore KD, Schneider DA, Newman JW, Adams SH and Hwang DH Saturated fatty acids activate TLR-mediated proinflammatory signaling pathways. J Lipid Res 53: 2002-2013, 2012.

31. Ye L, Kleiner S, Wu J, Sah R, Gupta RK, Banks AS, Cohen P, Khandekar MJ, Boström P, Mepani RJ, et al: TRPV4 is a regulator of adipose oxidative metabolism, inflammation, and energy homeostasis. Cell 151: 96-110, 2012

32. Zeisel MB, Dhawan P and Baumert TF: Tight junction proteins in gastrointestinal and liver disease. Gut 68: 547-561, 2018.

33. Krischer J, Gilbert A, Gorden P and Carpentier JL: Endocytosis is inhibited in hepatocytes from diabetic rats. Diabetes 42 1303-1309, 1993.

34. Yang M, He RL, Benovic JL and Ye RD: beta-Arrestin1 interacts with the Gprotein subunits betalgamma2 and promotes beta1gamma2-dependent Akt signalling for NF-kappaB activation. Biochem J 417: 287-296, 2009.

35. Russell DW, Schneider WJ, Yamamoto T, Luskey KL, Brown MS and Goldstein JL: Domain map of the LDL receptor: Sequence homology with the epidermal growth factor precursor. Cell 37: 577-585, 1984

36. Zhang L, Fairall L, Goult BT, Calkin AC, Hong C, Millard CJ, Tontonoz P and Schwabe JW: The IDOL-UBE2D complex mediates sterol-dependent degradation of the LDL receptor. Genes Dev 25: 1262-1274, 2011.

37. Bordone L and Guarente L: Calorie restriction, SIRT1 and metabolism: Understanding longevity. Nat Rev Mol Cell Biol 6 : 298-305, 2005.

38. Brooks CL and Gu W: How does SIRT1 affect metabolism, senescence and cancer? Nat Rev Cancer 9: 123-128, 2009.

39. Chang HC and Guarente L: SIRT1 and other sirtuins in metabolism. Trends Endocrinol Metab 25: 138-145, 2014.

40. Scott RA, Lagou V, Welch RP, Wheeler E, Montasser ME, Luan J, Mägi R, Strawbridge RJ, Rehnberg E, Gustafsson S, et al: Large-scale association analyses identify new loci influencing glycemic traits and provide insight into the underlying biological pathways. Nat Genet 44: 991-1005, 2012.
41. Huyghe JR, Jackson AU, Fogarty MP, Buchkovich ML, Stančáková A, Stringham HM, Sim X, Yang L, Fuchsberger C, Cederberg $\mathrm{H}$, et al: Exome array analysis identifies new loci and low-frequency variants influencing insulin processing and secretion. Nat Genet 45: 197-201, 2013.

42. Hara K, Fujita H, Johnson TA, Yamauchi T, Yasuda K, Horikoshi M, Peng C, Hu C, Ma RC, Imamura M, et al: Genome-wide association study identifies three novel loci for type 2 diabetes. Hum Mol Genet 23: 239-246, 2014.

43. Higurashi M, Ohya Y, Joh K, Muraguchi M, Nishimura M, Terawaki H, Yagui K, Hashimoto N, Saito Y and Yamada K: Increased urinary levels of CXCL5, CXCL8 and CXCL9 in patients with Type 2 diabetic nephropathy. J Diabetes Complications 2009 23: 178-184, 2009.

44. Nawaz MI, Van Raemdonck K, Mohammad G, Kangave D, Van Damme J, Abu El-Asrar AM and Struyf S: Autocrine CCL2, CXCL4, CXCL9 and CXCL10 signal in retinal endothelial cells and are enhanced in diabetic retinopathy. Exp Eye Res 109: 67-76, 2013.

45. Zychowska M, Rojewska E, Pilat D and Mika J: The role of some chemokines from the CXC subfamily in a mouse model of diabetic neuropathy. J Diabetes Res 2015: 750182, 2015.

46. Yu J, Wu H, Liu ZY, Zhu Q, Shan C and Zhang KQ: Advanced glycation end products induce the apoptosis of and inflammation in mouse podocytes through CXCL9-mediated JAK2/STAT3 pathway activation. Int J Mol Med 40: 1185-1193, 2017.

47. Melander O, Maisel AS, Almgren P, Manjer J, Belting M, Hedblad B, Engström G, Kilger U, Nilsson P, Bergmann A and Orho-Melander M: Plasma proneurotensin and incidence of diabetes, cardiovascular disease, breast cancer, and mortality. JAMA 308: 1469-1475, 2012.

48. Li J, Song J, Zaytseva YY, Liu Y, Rychahou P, Jiang K, Starr ME, Kim JT, Harris JW, Yiannikouris FB, et al: An obligatory role for neurotensin in high fat diet-induced obesity. Nature 533: 411-415, 2016.

49. Léon C, Freund M, Latchoumanin O, Farret A, Petit P, Cazenave JP and Gachet $\mathrm{C}$ : The $\mathrm{P} 2 \mathrm{Y} 1$ receptor is involved in the maintenance of glucose homeostasis and in insulin secretion in mice. Purinergic Signal 2005: 145-151, 2005.

50. Breuker C, Amouzou C, Fabre O, Lambert K, Seyer P, Bourret A, Salehzada T, Mercier J, Sultan A and Bisbal C: Decreased RNF41 expression leads to insulin resistance in skeletal muscle of obese women. Metabolism 83: 81-91, 2018.

51. Pearson G and Soleimanpour SA: A ubiquitin-dependent mitophagy complex maintains mitochondrial function and insulin secretion in beta cells. Autophagy 14: 1160-1161, 2018.

This work is licensed under a Creative Commons Attribution-NonCommercial-NoDerivatives 4.0 International (CC BY-NC-ND 4.0) License. 